\title{
Clinical usefulness of autoantibodies in idiopathic inflammatory myositis
}

\author{
Ignacio García-De La Torre* \\ Department of Immunology and Rheumatology, Hospital General de Occidente de la Secretaría de Salud, University of \\ Guadalajara, Zapopan, México
}

Keywords: autoantibodies, inflammatory myositis, clinical utility of autoantibodies, myositis-specific antibodies, myositis-associated antibodies

\section{Introduction}

One of the most important characteristics of systemic autoimmune diseases, including idiopathic inflammatory myopathies (IIM), is the immune response to self-antigens manifested by the production of autoantibodies that recognize a variety of cytoplasmic and nuclear antigens. In the last 40 years, autoantibodies of patients with IIM have been investigated; however, some of the fundamental questions related to these antibodies remain unresolved (1).

Indirect immunofluorescence (IIF), in which HEp-2 cells are used as a substrate, is considered the gold standard for detecting antinuclear antibodies (ANA's) in autoimmune rheumatic diseases. Using this method, specific antibodies that occur exclusively in these diseases were present in a great proportion of patients with myositis (50-80\%) (2). It is important to note that patients with myositis more frequently have high titers of these antibodies than patients with overlap syndromes, and low titers usually are present in patients with myopathies associated with neoplasia. In contrast, these autoantibodies are usually absent in patients with inclusion body myositis, dystrophies, and other non-autoimmune myopathies.

In general, even though the diagnostic value of these autoantibodies has been limited, they are useful as diagnostic markers since they closely correlate with certain clinical manifestations of disease and help identify clinical subgroups and to know about the prognosis of the IIM.

Idiopathic inflammatory myopathies has two major types of autoantibody groups: (1) those found primarily in patients suffering from polymyositis (PM) or dermatomyositis (DM) are known as myositis-specific autoantibodies (MSAs); these are not observed in other diseases of connective tissue and are almost completely absent in patients with muscular dystrophies and (2) those that appear in myositis overlap syndromes and in other connective tissue diseases, which correlate with certain clinical and/or pathophysiological conditions of myositis, are known as myositis-associated autoantibodies (MAAs) (3).

\section{Myositis-Specific Autoantibodies}

In general, MSAs are useful markers for clinical diagnosis, classification, and for predicting the prognosis of the IIM. MSAs have two major autoantibody subsets. Probably, the largest and most interesting subset is the anti-cytoplasmic antibodies, which consist of two main groups: those antibodies that are directed to various aminoacyl-tRNA synthetases (ARSs), cytoplasmic enzymes responsible for catalyzing the esterification of a specific amino acid to its cognate tRNA, forming an aminoacyl-tRNA. To each of the 20 amino acids corresponds a unique tRNA.

Autoantibodies against histidyl-tRNA-synthetase (anti-Jo-1) (4) are the most common MSAs that belong to anti-cytoplasmic antibodies and were first described in 1980.These antibodies are found in approximately $25-30 \%$ of myositis patients, and they attach histidine to the appropriate tRNA. The anti-Jo-1 antibodies (4) were subsequently found in a group of patients with anti-synthetase 
syndrome (5), a unique clinical syndrome that includes myositis, interstitial lung disease (ILD), non-erosive arthritis, fever, and characteristic hyperkeratotic lesions along the radial and palmar aspects of the fingers known as "mechanic's hands."

Other antibodies from the same subgroup of anti-cytoplasmic antibodies targeting a number of additional ARSs have since been identified, including those recognizing threonyl-tRNAsynthetase (anti-PL-7), alanyl-tRNA synthetase (anti-PL-12), glycyl-tRNA synthetase (anti-EJ), isoleucyl-tRNA synthetase (anti-OJ), asparaginyl-tRNA synthetase (anti-KS), tyrosyl-tRNA synthetase (anti-YRS), and, most recently, phenylalanyl synthetase (anti-Zo) (4). This group of autoantibodies to ARS occurs in approximately $1-5 \%$ of patients with myositis (5). It is worth noting that antibodies to one synthetase do not appear to crossreact with other synthetases, but other autoantibodies may occur with anti-synthetase, such as anti-SS-A and anti-SS-B.

The other subsets of anti-cytoplasmic antibodies are the antisignal-recognition particle antibodies (anti-SRP). This is a nonsynthetase antibody, and is found in approximately $4 \%$ or less of patients with myositis. SRP is a six-polypeptide complex (72, $68,54,19,14$, and $9-\mathrm{kDa}$ ) and one 7SL RNA molecule. The cytosolic ribonucleoprotein binds to the endoplasmic reticulum (ER), signaling sequences of elongating polypeptide chains during the synthesis and translocating them to the ER membrane. This autoantibody was first described in 1986 in a "typical PM" patient (6).

Later work showed that autoantibodies could be directed to any of the six polypeptides, as well as to 7SL RNA. The clinical features in the majority of patients with anti-SRP antibodies are unusually severe muscle weakness, dysphagia, and high creatinine kinase levels; poor response to steroids; and a worse prognosis compared to that in patients with other myositis. Furthermore, a large amount of necrotic and regenerating fibers were found in muscle biopsies taken from these patients, who also showed a lower frequency of lymphocytic inflammation than patients affected by other myositis (7).

The other group of MSAs is ANA, which occurs more frequently (approximately 60\%) than anti-cytoplasmic antibodies, and it shows a homogeneous, speckled, or nucleolar pattern. AntiMi-2 is the only ANA that is also considered a MSA and was first described in 1976 (8). In 1995, their cognate antigens were cloned and autoantibodies to $\mathrm{Mi}-2$ immunoprecipitated a nuclear complex consisting of up to eight subunits. Currently there are two known protein targets, Mi-2 $\alpha(240-\mathrm{kDa})$ and $\mathrm{Mi}-2 \beta(218-\mathrm{kDa})$, of the Mi-2 antigen.

Both forms of Mi-2 are different molecules that contain a set of helicase motifs with a similar function as nuclear helicases. Mi-2 $\beta$ and histone deacetylases form a protein complex called nucleosome-remodeling deacetylase complex, (9) which may be involved in gene transcription through histone acetylation, causing a remodeling of the nucleosome structure. In most studies, an antibody to Mi-2 has been associated with DM in adult and juvenile patients. It is rarely present in PM and leads to fewer complications related to ILD. In general, patients who have Mi-2 have a good prognosis and show good response to therapy.

A number of other MSAs have been described in different populations of patients with myositis. Clinical usefulness of these autoantibodies has been identified in patients with amyopathic DM and malignant-associated myositis.

In 2005, a specific autoantibody was identified in patients with clinically amyopathic DM (C-ADM), which is a term suggested for patients with typical DM rashes for at least 2 years, but without muscle symptoms. In the early 1990s, these patients were considered to have DM sine myositis. This specific antibody was detected in eight of 15 patients (53\%) with C-ADM by immunoprecipitation and immunoblotting. This antibody was termed anti-CADM140 , since it precipitated a $140-\mathrm{kDa}$ protein.

Typical DM rashes were found in the eight patients who had anti-CADM-140 in this study; however, they showed no muscle weakness or muscular pain and had normal serum creatinine kinase levels. In another study of 15 patients who had C-ADM, 13 of them developed ILD; of these, five developed an acute progressive type of ILD. Four of these patients had anti-CADM140. Therefore, antibody to CADM-140 may serve as a specific serologic marker for patients with myositis and acute ILD (10).

In subsequent studies, the target antigen of CADM-140 was identified using HeLa cell-derived complimentary DNA (cDNA) library. Specifically, the antibody reacted with the "melanoma differentiation-associated gene 5" (MDA-5) which encodes an RNA helicase that plays important roles in the innate immune system during RNA virus infection. The protein was expressed in cells transfected with full-length MDA-5 cDNA, confirming the identity of $M D A-5$ as the CADM-140 autoantigen.

In addition, an ELISA using recombinant $M D A-5$ protein as the antigen showed an analytical sensitivity of $85 \%$ and analytical specificity of $100 \%$ compared with the results of the "gold standard" immunoprecipitation assay. The antigen was also useful for identifying patients with $\mathrm{C}-\mathrm{ADM}$ and/or rapidly progressive ILD (11). By using indirect IIF, this antibody shows granular or reticular cytoplasmic staining resembling cytoskeletal components.

Targoff et al. described a previously unknown autoantibody that recognizes a $155-\mathrm{kDa}$ protein (p155) in patients with DM. This antibody is present mainly in patients with adult and juvenile DM and, interestingly, in patients with cancer-associated DM. Since it was present only in one patient with systemic lupus erythematosus (SLE) among a large group of non-myositis controls, this is considered a DM-specific antibody (12).

In another study, Targoff determined that the transcriptional intermediary factor $1-\gamma$ (TIF1- $\gamma$ ) is the target antigen of antip155 antibody. Interestingly, intracytoplasmic systems of protein translation are the primary immunological targets of PM-specific autoantibodies, while the targets of DM-specific autoantibodies are nuclear transcription factors. This suggests that PM and DM have different pathogenic mechanisms or different production systems of MSAs.

A Japanese group studying 52 myositis patients found an autoantibody that immunoprecipitated 155 - and $140-\mathrm{kDa}$ proteins in seven of them (13\%). These patients (anti-p155/p140positive) showed more frequent typical DM rashes than the other patients, but none had ILD. This autoantibody seems to be the same as the autoantibody to p155 reported by Targoff et al. However, the Japanese group claims they are not the same, since the $155-\mathrm{kDa}$ band was always associated with a $140-\mathrm{kDa}$ band in their study. 
TABLE 1 | Autoantibodies in myositis, their target antigens, M.W., frequency, and clinical significance.

\begin{tabular}{|c|c|c|c|c|}
\hline Antibodies & Nature of target antigens & $\begin{array}{l}\text { MW of antigens } \\
\text { in } k D a\end{array}$ & $\begin{array}{l}\text { Frequency } \\
\text { In IIM (\%) }\end{array}$ & Clinical significance \\
\hline \multicolumn{5}{|c|}{ MYOSITIS-SPECIFIC AUTOANTIBODIES (MSAS) } \\
\hline \multicolumn{5}{|c|}{ anti-ARS } \\
\hline anti-Jo-1 & Histidyl-tRNA synthetase & $50 / 52$ & $15-20$ & \multirow{8}{*}{$\begin{array}{l}\text { Antisynthetase syndrome (myositis, interstitial lung } \\
\text { disease, polyarthritis, mechanic's hand Raynaud's } \\
\text { phenomenon, fever) }\end{array}$} \\
\hline anti-PL-7 & Threonyl-tRNA synthetase & 83 & $5-10$ & \\
\hline anti-PL-12 & Alanyl-tRNA synthetase & 70 & $<5$ & \\
\hline anti-EJ & Glycyl-tRNA synthetase & 75 & $5-10$ & \\
\hline anti-OJ & Isoleucyl-tRNA synthetase & 145 & $<5$ & \\
\hline anti-KS & Asparaginyl-tRNA synthetase & 63 & $<5$ & \\
\hline anti-Zo & Phenylalanyl-tRNA synthetase & 52 & $<1$ & \\
\hline anti-YRS & Tyrosyl-tRNA synthetase & 59 & $<1$ & \\
\hline anti-SRP & Signal recognition particle & 9-72 & $<4$ & Necrotizing myopathy \\
\hline anti-Mi-2 & Mi-2 $\alpha / \mathrm{Mi}-2 \beta$ helicase family proteins & $240 / 218$ & $5-10$ & Dermatomyositis \\
\hline anti-CADM-140 & Melanoma differentiation-associated gene 5 (MDA-5) & 140 & 50 (C-ADM) & Specific in C-ADM and acute ILD \\
\hline Anti-p155(/p140) & Transcriptional intermediary factor $1 \gamma($ TIF1- $\gamma)$ & $155 / 140$ & $20(\mathrm{DM})$ & $\begin{array}{l}\text { Dermatomyositis, especially in cancer-associated } \\
\text { dermatomyositis }\end{array}$ \\
\hline anti-MJ & Nuclear matrix protein (NXP-2) & 140 & $<23$ & Juvenile dermatomyositis \\
\hline anti-PMS1 & DNA repair mismatch enzyme & 120 & 7.5 & DM and PM \\
\hline anti-SAE & Small ubiquitin-like modifier activating enzyme & $40 / 90$ & $<4$ & Dermatomyositis \\
\hline anti-HMGCR & HMG-CoA reductase & $200 / 100$ & $62 \%$ & Statin-induced autoimmune-necrotizing myopathy \\
\hline \multicolumn{5}{|c|}{ MYOSITIS-ASSOCIATED AUTOANTIBODIES (MAAS) } \\
\hline anti-U1RNP & U1 small nuclear RNP & $11-70$ & 10 & Overlap myositis, MCTD \\
\hline anti-SS-A/Ro & Ro-52/TRIM21 and Ro-60 proteins & $52 / 60$ & $\geq 35 \%$ & ILD in IIM patients \\
\hline anti-Ku & DNA-PK regulatory subunit & $70 / 80$ & $\overline{20}-30$ & Polymyositis-SSc overlap in Japanese \\
\hline anti-PM-Scl & Nucleolar protein complex of $11-16$ proteins & $75 / 100$ & $10-15$ & Polymyositis-SSc overlap \\
\hline
\end{tabular}

ARS, aminoacyl-tRNA synthetases; C-ADM, clinically amyopathic dermatomyositis; ILD, interstitial lung disease; HMGCR, 3-hydroxy-3 methylglutaryl-coenzyme A reductase; DNA-PK, DNA-dependent protein kinase; MCTD, mixed connective tissue disease; SSc-scleroderma. MW, molecular weight; kDa, kilodalton.

A preliminary study of United States patients with juvenile DM described a new anti-MJ antibody that targets a $140-\mathrm{kDa}$ protein. It was identified as nuclear matrix protein NXP-2 (13). Moreover, Gunawardena et al., studying UK patients with juvenile DM, found an autoantibody to a $140-\mathrm{kDa}$ protein that was identical to the anti-MJ (anti-NXP-2) antibody. They screened 162 juvenile myositis patients and found $23 \%$ of them positive for anti-NXP2 antibody. The autoantibody was detected only in patients with juvenile DM. It has been confirmed that this antibody is different from anti-CADM-140 and anti-p155.

Betteridge et al. (14) reported a new autoantibody against small ubiquitin-like modifier activating enzyme (SAE) in patients with DM. The post-translational modification of specific proteins such as protein kinases and transcription factors is greatly influenced by small ubiquitin-like modifiers. The authors studied 266 patients with myositis, 250 with other rheumatic diseases, and 50 healthy controls. Of these, 11 (4\%) had anti-SAE, which immunoprecipitated both 40 and $90 \mathrm{kDa}$ proteins; all of these patients suffered from DM and showed classical skin lesions (13).

A new autoantibody associated with necrotizing myopathy was also described by Christopher-Stine et al. in a preliminary report. The authors studied immunoprecipitations from ${ }^{35} S$-methioninelabeled HeLa cell lysates of 225 patients with myositis and reported necrosis on muscle biopsy in 38 patients but no perifascicular atrophy or red-rimmed vacuoles; 26 patients showed unidentified MSAs (15).

In 16 of these 26 patients, an anti-200/100 antibody, which immunoprecipitated both 200 -and $100-\mathrm{kDa}$ proteins, was found. In subsequent studies, the $100-\mathrm{kDa}$ autoantigen was identified as the 3-hydroxy-3-methylglutaryl-coenzyme A reductase
(HMGCR), the pharmacologic target of statin medications. Additional experiments indicated that the $200-\mathrm{kDa}$ autoantigen is the dimeric form of HMGCR (15).

The majority of these patients (63\%) had a history of exposure to statins prior to developing muscle weakness. It is not clear if exposure to this drug intensifies this myopathy, but these results may provide useful information on the immune-mediated mechanisms of necrotizing myopathy.

A list of these MSAs and MAAs is outlined in Table $\mathbf{1 .}$

\section{Myositis-Associated Autoantibodies}

The more common MAAs includes anti-U1-RNP, anti-SS-A/Ro, anti-PM/Scl, and anti-Ku, which are found in patients with myositis and various overlap syndromes (16).

$\mathrm{U} 1-\mathrm{RNP}$ is a small nuclear ribonucleoprotein consisting of U1-snRNA and nine proteins: $70 \mathrm{~K}(70-\mathrm{kDa}), \mathrm{A}(34-\mathrm{kDa})$, B'/B (29/28-kDa), C (22-kDa), D (16-kDa), E (13-kDa), F $(12-\mathrm{kDa})$, and $\mathrm{G}(11-\mathrm{kDa})$. It is part of a spliceosome that eliminates intervening sequences from pre-mRNA to produce mature mRNA. Anti-U1-RNP antibody is a serological marker for mixed connective tissue disease, very common in definite scleroderma (SSc), SLE, PM/DM, and overlapping syndromes.

Anti-SS-A/Ro antibody is found in some autoimmune diseases such as Sjögren's syndrome, SLE, SSc, and IIM. AntiSS-A/Ro recognizes two distinct antigens, with molecular weights of $52-\mathrm{kD}$ (Ro-52/TRIM21) and 60-kDa (Ro-60), respectively. Each antibody has a different clinical association. Anti-Ro-52/TRIM21 is significantly more prevalent in SSc and 
myositis than anti-Ro-60, while isolated anti-Ro-52/TRIM21 may be found in up to $37 \%$ of patients with myositis, often in correlation with anti-Jo-1 reactivity. The presence of anti-SSA/Ro-52/TRIM21 antibodies is associated with more severe ILD, particularly in patients with anti-synthetase syndrome (13).

Anti-PM/Scl antibodies are a heterogeneous group of autoantibodies directed to several proteins of the nucleolar $\mathrm{PM} / \mathrm{Scl}$ macromolecular complex. PM/Scl-75 and PM/Scl-100 are the two main autoantigenic protein components and are named based on their apparent molecular weights. These antibodies are associated with PM-SSc overlap syndrome in approximately $15 \%$ of the patients, and may be present also in other autoimmune diseases (16).

The antibody to $\mathrm{Ku}$ was reported for the first time in Japanese patients suffering from PM-SSc overlap syndrome, and it was considered a serologic marker of it. However, studies carried out in other countries have yielded different results. In the United States, anti-Ku antibody was found mostly in SLE patients. The difference in the results may be due to the use of different assay systems and the differences in ethnic or genetic background of the patients studied (16). The target antigen of anti-Ku antibody is a heterodimer of 70 - and $80-\mathrm{kDa}$ proteins that is thought to be

\section{References}

1. Reichlin M, Mattioli M. Description of a serological reaction characteristic of polymyositis. Clin Immunol Immunopathol (1976) 5:12-20. doi:10.1016/ 0090-1229(76)90145-8

2. Kavanaugh A, Tomar R, Reveille J, Solomon DH, Homburger HA. Guideline for the clinical use of the antinuclear antibody test and tests for specific autoantibodies to nuclear antigens. Arch Pathol Lab Med (2000) 124:71-81. doi:10.1043/0003-9985(2000)124<0071;GFCUOT>2.0CO:2

3. Mimori T, Imura Y, Nakashima R, Yoshifuji H. Autoantibodies in idiopathic inflammatory myopathy: an update on clinical and pathophysiological significance. Curr Opin Rheumatol (2007) 19:523-9. doi:10.1097/BOR. 0b013e3282f01a8c

4. Nishikai M, Reichlin M. Heterogeneity of precipitating antibodies in polymyositis and dermatomyositis. Characterization of the Jo-1 antibody system. Arthritis Rheum (1980) 23:881-8. doi:10.1002/art.1780230802

5. Love LA, Leff RL, Fraser DD, Targoff IN, Dalakas M, Plotz PH, et al. A new approach to the classification of idiopathic inflammatory myopathy: myositisspecific autoantibodies define useful homogeneous patient groups. Medicine (1991) 70:360-74. doi:10.1097/00005792-199111000-00002

6. Targoff IN, Johnson AE, Miller FW. Antibody to signal recognition particle in polymyositis. Arthritis Rheum (1990) 33:1361-70. doi:10.1002/art.1780330908

7. Miller T, Al-Lozi MT, Lopate G, Pestronk A. Myopathy with antibodies to the signal recognition particle: clinical and pathological features. J Neurol Neurosurg Phychiatry (2002) 73:420-8. doi:10.1136/jnnp.73.4.420

8. Targoff IN, Reichlin M. The association between $\mathrm{Mi}-2$ antibodies and dermatomyositis. Arthritis Rheum (1985) 28:796-803. doi:10.1002/art. 1780280711

9. Zhang Y, LeRoy G, Seelig HP, Lane WS, Reinberg D. The dermatomyositisspecific autoantigen $\mathrm{Mi}-2$ is a component of a complex containing histone deacetylase and nucleosome remodeling activities. Cell (1998) 95:279-89. doi:10.1016/S0092-8674(00)81758-4

10. Sato S, Hirakata M, Kuwana M, Suwa A, Inada S, Mimori T, et al. Autoantibodies to a 140-kd polypeptide, CADM-140, in Japanese patients with clinically an activation subunit of DNA-dependent protein kinase. The $\mathrm{Ku}$ antigen has key roles in various cellular processes, including DNA double-strand break repair.

\section{Conclusion}

In the last 20 years, several MSAs and MAAs, and their target autoantigens, clinical phenotypes, and histological features have been identified. This group of autoantibodies may yield useful insights into their diagnosis, prognosis, and treatments.

Testing for the majority of these autoantibody specificities is already commercially available through different immunoassays. These methods aid to establish the correct diagnosis in cases in which an autoimmune muscle disease is suspected.

\section{Acknowledgments}

This work was supported in part by Programa Nacional de Posgrados de Calidad (PNPC) del Consejo Nacional de Ciencia y Tecnología (CONACyT), México city, and Universidad de Guadalajara, Guadalajara, México. Dr. IGDLT is investigator level II (SNI-II) of the National Research System of CONACyT.

amyophatic dermatomyositis. Arthritis Rheum (2005) 52:1571-6. doi:10.1002/ art. 21023

11. Sato S, Hoshino K, Satoh T, Fujita T, Kawakami Y, Fujita T, et al. RNA helicase encoded by melanoma differentiation-associated gene 5 is a major autoantigen in patients with clinically amyopathic dermatomyositis: association with rapidly progressive interstitial lung disease. Arthritis Rheum (2009) 60:2193-200. doi:10.1002/art.24621

12. Targoff IN, Mamyrova G, Trieu EP, Perurena O, Koneru B, O’Hanlon TP, et al. A novel autoantibody to a $155-\mathrm{kd}$ protein is associated with dermatomyositis. Arthritis Rheum (2006) 54:3682-9. doi:10.1002/art.22164

13. Nakashima R, Mimori T. Clinical and pathophysiological significance of myositis-specific and myositis-associated autoantibodies. Int J Clin Rheumtol (2010) 5:523-36. doi:10.2217/ijr.10.48

14. Betteridge ZE, Gunawardena H, Chinoy H, North J, Ollier WE, Cooper RG, et al. Clinical and human leucocyte antigen class II haplotype associations of autoantibodies to small ubiquitin-like modifier enzyme, a dermatomyositisspecific autoantigen target, in UK Caucasians adult-onset myositis. Ann Rheum Dis (2009) 68:1621-5. doi:10.1136/ard.2008.097162

15. Casciola-Rosen L, Mammen AL. Myositis autoantibodies. Curr Opin Rheumatol (2012) 24:602-8. doi:10.1097/BOR.0b013e328358bd85

16. Lega JC, Fabien N, Reynaud Q, Durieu I, Durupt S, Dutertre M, et al. The clinical phenotype associated with myositis-specific and associated autoantibodies: a meta-analysis revisiting the so-called antisynthetase syndrome. Autoimmun $\operatorname{Rev}(2014)$ 13:883-91. doi:10.1016/j.autrev.2014.03.004

Conflict of Interest Statement: The author declares that this manuscript and associated research was done in the absence of any commercial or financial relationships that could be construed as a potential conflict of interest.

Copyright (C) 2015 García-De La Torre. This is an open-access article distributed under the terms of the Creative Commons Attribution License (CC BY). The use, distribution or reproduction in other forums is permitted, provided the original author(s) or licensor are credited and that the original publication in this journal is cited, in accordance with accepted academic practice. No use, distribution or reproduction is permitted which does not comply with these terms. 